\title{
Developing a Model for Assessment of Traffic Distraction
}

\author{
Mia Suhanek*, Ivan Djurek, Sanja Grubesa, Antonio Petosic \\ Department of Electroacoustics, Faculty of EE and Computing, University of Zagreb, Zagreb, Croatia
}

Email address:

mia.suhanek@fer.hr (M. Suhanek)

${ }^{*}$ Corresponding author

\section{To cite this article:}

Mia Suhanek, Ivan Djurek, Sanja Grubesa, Antonio Petosic. Developing a Model for Assessment of Traffic Distraction. American Journal of Traffic and Transportation Engineering. Vol. 3, No. 3, 2018, pp. 50-56. doi: 10.11648/j.ajtte.20180303.12

Received: July 10, 2018; Accepted: July 20, 2018; Published: August 17, 2018

\begin{abstract}
The soundscape of an expressway in Zagreb was recorded and reproduced to two groups of listeners while they were performing a concentration demanding task - a simplified variation of the memory game. This expressway was chosen due to the high traffic frequency and on the other hand, due to the large number of residential buildings that are near the expressway. The focus of the research is whether the residents of a large city, in their daily life have adapted to this noise and how. We also wanted to investigate what is the main reason of their annoyance and distraction. Furthermore, we wanted to quantify human distraction with this typical urban soundscape by developing and then using the acoustic model for total distraction level.
\end{abstract}

Keywords: Soundscape, Traffic Noise, Loudness, Distraction, Acoustic Model

\section{Introduction}

The initial concept of soundscape was proposed as an attempt to create an analytical perspective that would explain the total acoustic environment over time and across cultures [1]. Most soundscape studies concern the qualitative analysis of soundscapes; however, the methods for evaluating a soundscape vary depending on the purposes of the studies and the researchers conducting them [2,3]. Taking into account several variables such as loudness, pitch strength and fluctuation of pitch strength among others, it is possible to calculate the annoyance level of a certain sound or a soundscape [4-6]. Another part of soundscape and sound analysis deals with their distraction properties and the way in which characteristic sounds influence people performing logical, mathematical and other concentration demanding tasks [7, 8].

People are exposed to different sound environments on a daily basis. Acoustic modellingin terms of soundscape is nowadays very important due to ever increasing noise pollution especially in urban areas. Long exposure to a specific sound environment results in eventual adaptation to that environment, and small and expected loudness changes in soundscape do not significantly influence one's perception of that soundscape [9]. Frequent exposure to even a slight change in our everyday sound environment would also result in adaptation; furthermore, providing the loudness levels were not significantly higher, these changes would not be perceived as annoying [10].

Numerous studies dealing with human performance and perception under different sound and noise conditions usually consist of listening to certain sound stimuli and performing various tasks in a specific work environment [7, 8]. Most research to date focuses on the way different sounds influence listener's perception and distraction, and involves short artificial sounds, mainly noise, with different spectral content, duration and level [11-13]. Recent research established that loudness change and the rate of loudness change of artificial sounds both have a substantial influence on disturbance and annoyance perception [14]. On the other hand, listening tests involving natural sounds were limited to measuring overall acoustical quality and annoyance level, and not determining certain qualitative parameters such as duration, spectral content, etc [9].

In [15] we have analysed four different soundscapes and established the methodology for subjective evaluation of typical urban acoustic environments. In this paper, we applied similar methodology to assess how traffic noise influences human concentration and ability to perform every day activities. To do this, we have analysed a soundscape of typical city artery (in particular, the expressway called Ljubljanska Avenue, Zagreb, Croatia) in terms of total 
distraction level, which can be considered as an extension of research in [15]. We also wanted to analyse and compare in what way a soundscape of an expressway with different loudness distributions distract listeners performing a rather simple but concentration-demanding task, like a simplified variation of the memory game. The test subjects were only given technical information pertaining to the testing environment, and received no other training that could disturb the results of the test. The specific game was chosen due to the subjects' familiarity with its format.

Furthermore, the level of concentration required, for this simplified variation of the game, corresponds to day-to-day situations where people perform basic tasks while being exposed to different sounds which do not require their focus, but only cause distraction.

The core objective of this research was to monitor listeners' reactions to different sounds in the background of the main soundscape theme and to determine whether these sound events cause distraction on the same level as louder, familiar and expected sounds of soundscape.

The underlying idea was that the listeners perform a certain task for the duration of the soundscape and perceive the soundscape subconsciously, rather than actually listen to the recording. The results of the game for all listeners were compared to questionnaire responses which lead to the conclusion that, when analysing soundscape in general, listeners base the level of their annoyance mainly on loudness. On the other hand, distraction can also be attributed to unexpected sounds that are somewhat different from the main sound print. Furthermore, using the model for total annoyance an overall quantitative value of total annoyance is calculated for this specific acoustic environment.

\section{The Soundscape of an Expressway}

The expressway soundscape was recorded using the soundwalk method [3, 16, 17] and is characterized by two easily discernible parts: the steady part and the variable part (Figure 1). In both cases the average reproduced sound pressure level in the steady part of the recording was relatively low $-50 \mathrm{~dB}(\mathrm{~A})$, corresponding to loudness of about 4 sones[4, 5]. Loudness versus time diagram is shown in Figure 2. Ljubljanska Avenue is an expressway stretching from the east to the western exit of the Croatian capital Zagreb (Figure 3). Traffic in this avenue is almost always heavy and dense so the soundscape included sounds coming from cars, buses and trucks passing by, audible traffic signal for the visually impaired et cetera. An average person living in an urban setting would be familiar with that kind of soundscape.

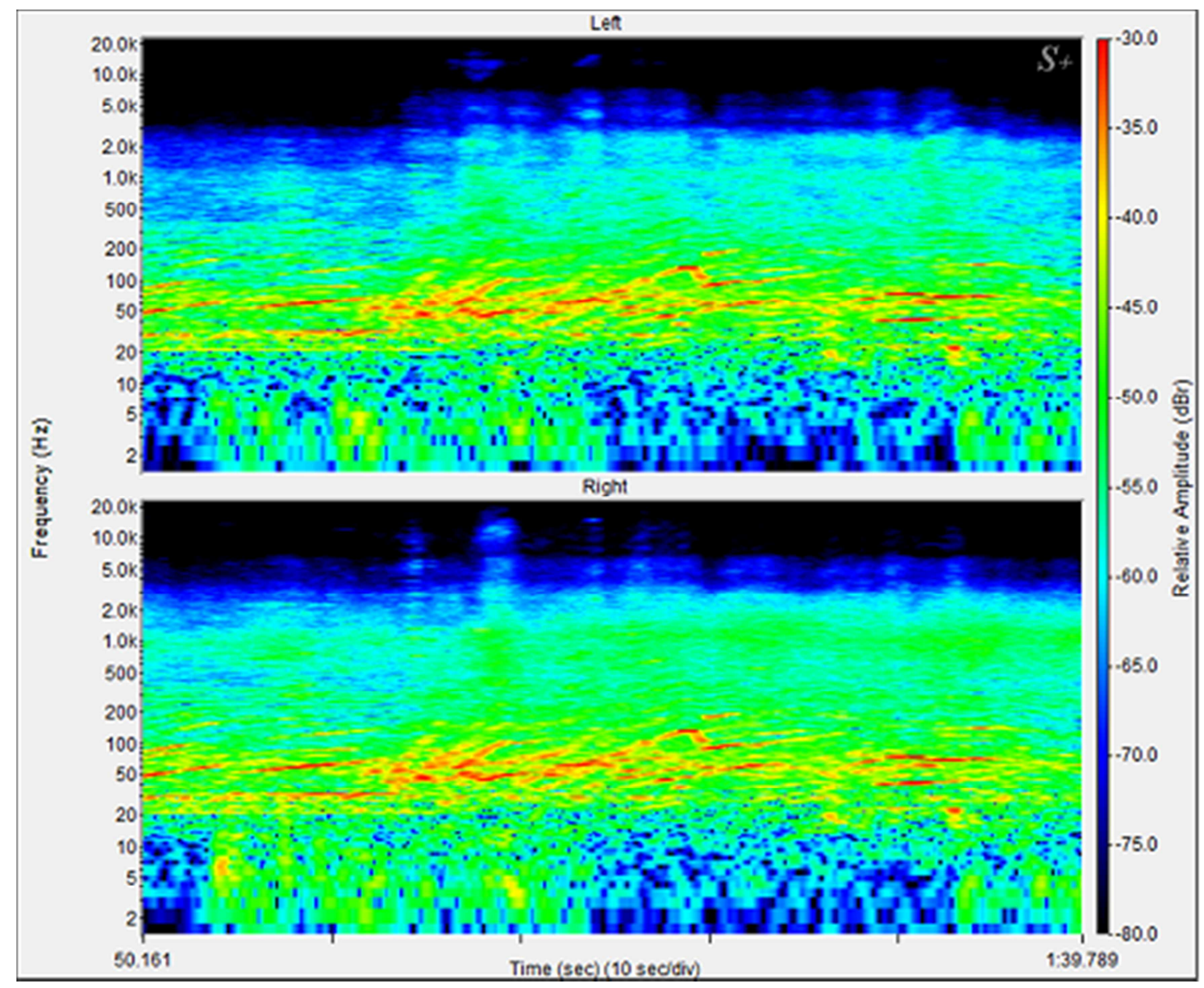

Figure 1. Spectrogram of the expressway. 


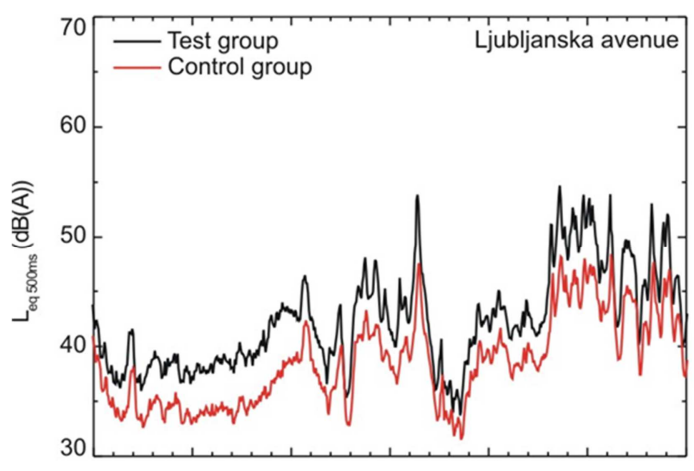

Figure 2. Loudness versus time diagram for control and experimental group.

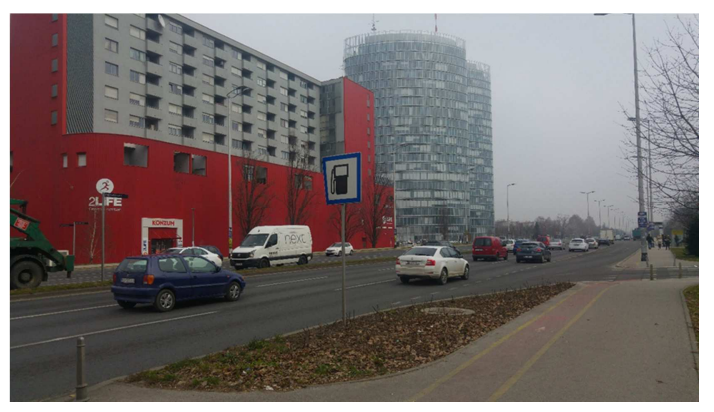

Figure 3. The expressway- view to east.
We wanted to give the test subjects time to adapt to a certain soundscape; however, our previous practical experiences with exposing test subjects to similar sound stimuli for longer than 10 minutes, proved to cause fatigue in test subjects. Therefore, the duration of soundscape was chosen to be around 7 minutes since we believe this interval to be optimal for adapting to a new sound environment and, at the same time, retaining attention [22]. Sudden and large loudness changes did not occur during the first minute of a soundscape. The steady part of a soundscape enabled listeners to mainly concentrate on the game, as they would on any task of similar complexity in their everyday routine.

The listeners were divided into two groups: control and experimental. The experimental group listened to the soundscape with sudden and short loudness changes, that is, without any post-processing modifications. The control group listened to the same soundscape but with narrower loudness distribution over the entire length of the recordings. This was achieved through dynamic post-processing. For the control group of listeners, the soundscape was passed through a compressor in order to lower the maximum values of loudness; however, the average loudness retained the same values. For the control group soundscape, maximum loudness did not exceed 7 sones.

Table 1. The t-test $(\alpha=0.05)$ for the average score of experimental and control groups.

\begin{tabular}{|c|c|c|c|c|c|c|c|c|c|}
\hline \multicolumn{10}{|c|}{ Expressway } \\
\hline C.G. & & E.G. & & & & & & & \\
\hline $\mathbf{X}_{\text {avg }}$ & $\mathbf{n}_{\mathbf{x}}$ & $\mathbf{s}_{x}^{2}$ & $\mathbf{Y}_{\text {avg }}$ & $\mathbf{n}_{\mathbf{y}}$ & $\mathbf{s}_{y}^{2}$ & $X_{a v g}-Y_{a v g}$ & $\left|\mathbf{X}_{\text {avg }}-\mathbf{Y}_{\text {avg }}\right|$ & $\mathbf{S}_{\mathbf{X}-\mathbf{Y a}}$ & $\mathrm{t}($ for $\alpha=0.05 \mathrm{t}=2.01)$ \\
\hline 0.96 & 50 & 0.0017 & 0.93 & 50 & 0.0034 & 0.03 & 0.03 & 0.0100 & 2.99 \\
\hline
\end{tabular}

The experimental research methodology postulates the manipulation of one independent variable, in this case sudden and unexpected loudness changes, while other variables are kept constant for both the experimental group and the control group [18-20]. According to psychological methodology, the two mentioned groups represent independent samples on which statistical significance can be established. It is important to emphasize the controlled aspects of the testing. The subject is not aware in which of the two groups is he or she placed nor aware of the studies final objective (the null-hypothesis). All the subjects are tested under the same conditions. According to psychological methodology, the conditions must be followed so that a certain hypothesis can be tested without a subject bias or interference. Using statistical methods the hypothesis can then be proven or discarded [18].

Regarding the statistics, in this research two independent samples were taken, and their average score has been analysed so it was possible to use the t-test in order to confirm the null-hypothesis. Using the t-test the proposed hypothesis for a sample can be easily proven and then applied to a population. When using a two-sample t-test for the difference between means of small independent samples it is necessary to identify the null hypotheses and alternative hypothesis. The next step is to specify the level of significance $\alpha$ and to determine the degrees of freedom $N-1$, where $N$ is defined as the size of a sample. According to the level of significance and the degrees of freedom critical value is determined. After finding the standardized test statistic it is possible to make a decision to reject or fail to reject the null hypothesis [21]. The so-called $t$-value which is the core of the $t$-test can be regarded as statistical equivalent of signal-to-noise ratio [22] and is calculated as:

$$
t=\frac{\bar{X}_{\text {exp }}-\bar{X}_{\text {cont }}}{\sqrt{\frac{\sigma_{\text {exp }}^{2}}{N_{\text {exp }}}+\frac{\sigma_{\text {cont }}^{2}}{N_{\text {cont }}}}}
$$

where $\bar{X}, \sigma^{2}$ and $N$ denote mean values of responses, variance and number of samples, respectively, while indices "exp" and "cont" refer to experimental and control group, respectively. Using standard table of significance [17] it is found that the postulated significance level of $\alpha=0.05$ corresponds to the demand for t-value to be greater than 2.01 in order for yield statistically significant difference between the mean values obtained for control and experimental group. Our hypothesis that there is a significant difference between the scores of experimental and control groups due to sudden and unexpected loudness changes is therefore proven (see Table $1)$.

Two groups of the same soundscape enabled us to identify one objective parameter, namely loudness. We wanted to determine whether individual sound events in the soundscape would be perceived by the control group of listeners as 
distracting if their loudness was of a lower value. This enabled us to extract individual sounds and analyse them separately.

\section{The Game}

The soundscape was reproduced to two groups of 50 listeners each; namely, the control and experimental groups. The experimental group listened to the soundscape with frequent sudden and short loudness changes, and the control group listened to the same soundscape but with lower loudness changes.

The recordings were reproduced using AKG K55 closed electrodynamic headphones with an average sound pressure level of $50 \mathrm{~dB}(\mathrm{~A})$ in the steady part of the recorded soundscape. Loudness was calculated using the established Zwicker method $[4,5]$ according to the norm DIN 45631. Free field equalization was used. The listeners' groups had equal female-to-male ratio, with median age of 24 . The listeners were not informed as to the content of the recording. The established psychological and statistical research methodology and praxis [18, 19, 20] was followed and respected in every way.

During the listening of a soundscape, the listeners had to solve an interactive game, similar to the traditional children's memory game, programmed in Matlab (Figure 4).

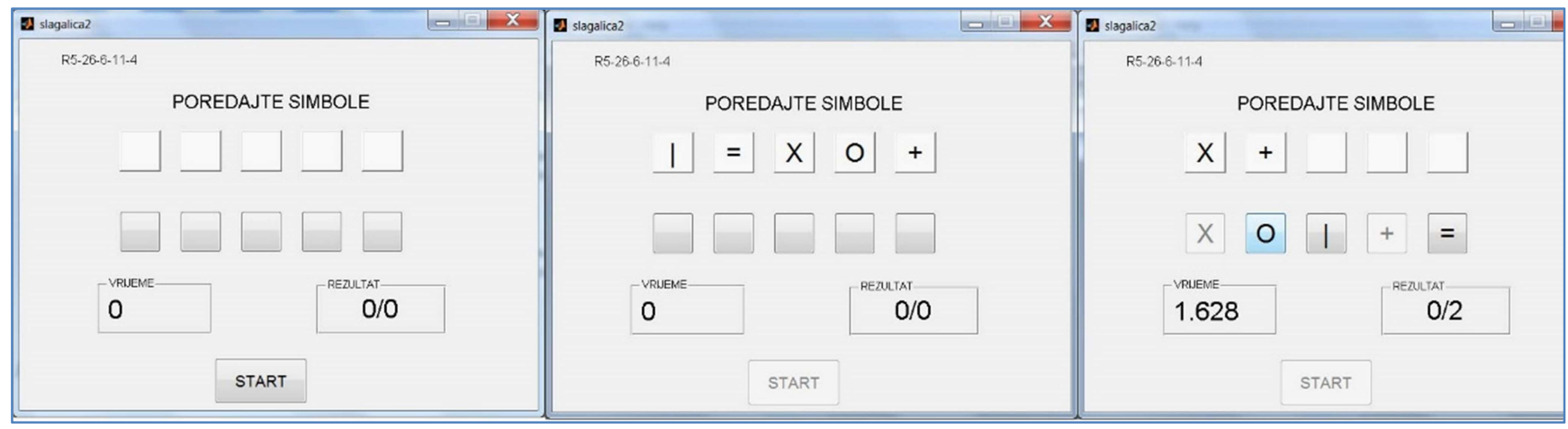

Figure 4. A snapshot of Memory game interface in Matlab.

The listeners played the game on a computer, while at the same time listening to a soundscape. The game uses five cards with simple mathematical symbols $(\mathrm{X}, \mathrm{O}, \mathrm{I},+,=)$. The symbols on the cards are revealed to the listener in a random order for four seconds. The goal is for the listener to line up the symbols on the cards according to the order in which they appeared. Time allotted for this task is seven seconds, with a two second pause before the next layout of the cards. If the listener makes a mistake while arranging the symbols on cards, a new hand of cards is drawn automatically. The game is not intended to be difficult or complicated, but rather to serve as means of assessing listener's concentration or the absence thereof during specific sound events in soundscape, i.e., sudden loudness changes. For the duration of a soundscape, the program generates orders of symbols and records the listener's score. In this way we could establish and analyse the potential correlation between the results of the game and time, as well as identify certain parts of a soundscape that could have caused the distraction occurring at a specific point in time. The results of the game, representing the success ratio of each move, are generated numerically. For instance, if a listener successfully arranged all the cards in the given time frame, their result for this move equalled 1. If a listener successfully arranged only 3 out of 5 cards, the result was $3 / 5=0.6$. We then calculated and compared the average scores overall.

Finally, for each listener, we created an average score versus time diagram for a soundscape and listening group. That was then used in calculating the overall average score versus time diagram. Figure 5 shows that diagram.

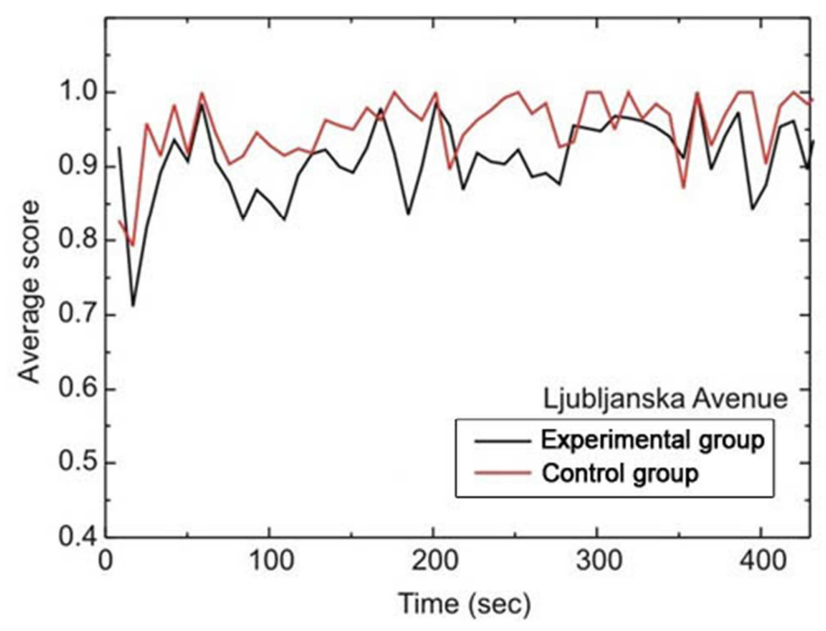

Figure 5. Expressway soundscape average game results versus time for experimental and control group.

A short questionnaire was composed in order to get a more detailed description of which particular sounds in a soundscape were the most annoying and why. The listeners had to provide an answer to a direct question: Which of the given sounds in the soundscape bothered you the most? This enabled us to establish a connection between annoying sounds and the results of the game. We supplied a list of potentially most annoying sounds in a soundscape. However, the list was far from selective as it contained all the characteristic sound events appearing in a soundscape. Our intention was to provide a reminder for the listeners considering the long duration of the soundscape sample, rather than to influence 
their answers. These results are laid out in Figure 6. In addition to the list of the characteristic sound events, the questionnaire provided the "all or nothing of the above" answer options.

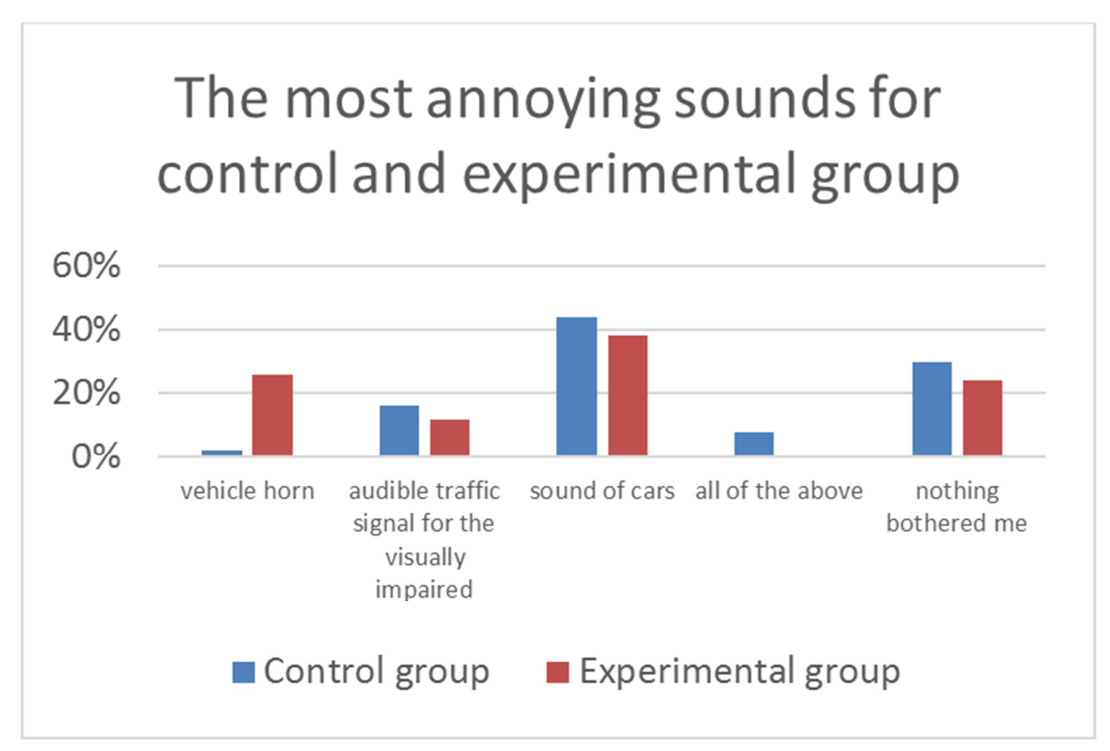

Figure 6. The most annoying sounds for the experimental and control groups.

\section{Discussion}

a. The game results

Upon careful examination the average move score tends to slightly increase toward the end of the soundscape (see Figure 5). Moreover, the first moves for all listeners are most inaccurate, while the average scores tend to increase toward the end of the soundscape. The reasons for this are twofold: adaptation to the game environment and adaptation to the soundscape. Despite the fact that all the listeners played some type of a memory game prior to this testing and were therefore familiar with the pattern of the game, their initial moves were considerably less accurate than those made later in the game. The recorded average move time stabilized after the first few moves and this can be attributed both to the adaptation to the game environment and adaptation to the soundscape factors.

There is an obvious difference for the experimental and control group soundscape in average score (Table 2). The difference in the average test results of the two groups obtained may, at first, seem statistically insignificant. However, using the $t$-test (with the significance level $\alpha=0.05$ ) it is shown that the results are, in fact, statistically significant (Table 1).

Table 2. The game results for the experimental and control groups.

\begin{tabular}{lll}
\hline & Expressway \\
\hline Group & EG & CG \\
Average score & 0.93 & 0.96 \\
Stand. deviation & 0.058 & 0.041 \\
The lowest score & 0.67 & 0.79 \\
$\mathrm{~N}_{5}$ (sone) $(7 \mathrm{~min})$ & 8 & 6.5 \\
\hline
\end{tabular}

b. The questionnaire results

The questionnaire responses describing the listeners' perception of the soundscape showed that, in the majority of cases, annoyance can be attributed to loud sounds - mainly those louder than the main theme of the soundscape (for the control group sound of cars and for the experimental group both sound of cars passing by and the sound of vehicle horn). On the other hand, a relatively large percentage of listeners stated that nothing bothered them. We can explain this; this soundscape consists mainly of the same and even more important; expected sounds, with very narrow loudness distributions.

c. Using the model of total distraction level

A linear model of total distraction level TDL [22] has been applied on this soundscape as an objective parameter. The model is defined with loudness difference $L_{D I F}$ (son), loudness growth rate difference $L G D_{D I F}$ (son/sec) and sharpness difference $\mathrm{S}_{\mathrm{DIF}}$ (acum) and all of these parameters are calculated for the expressway soundscape:

$$
\mathrm{TDL}=0.1 \cdot \mathrm{L}_{\mathrm{DIF}}+0.02 \cdot \mathrm{LGD}_{\mathrm{DIF}}+0.44 \cdot \mathrm{S}_{\mathrm{DIF}}
$$

The loudness difference is expressed in sones and is the most important parameter. The soundscape average sound loudness and the difference in loudness changes are calculated in Matlab. Then only positive values were taken and the loudness distribution function was calculated. The value $\Delta N_{5}$ is read from the loudness distribution function. The value $\Delta N_{5}$ is the level of the loudness difference that is "exceeded" for only $5 \%$ of the total time (or $95 \%$ of the time). A deviation factor from the mean loudness value is calculated using this data.

The difference in loudness growth rate is expressed in sons per second. It is taken as a parameter because it is shown as a significant parameter in the study with the short signals and also because it is expected that sudden loudness changes will cause greater annoyance. The average loudness growth rate and the difference in the rate of loudness increase changes over time are calculated. Only positive values are taken and the distribution function of the loudness growth rate difference 
is calculated. The $\triangle B P_{5}$ value is read out (the value of the loudness increase rate $\triangle B P_{5}$ is the level of the loudness increase rate which has been "exceeded" for only $5 \%$ of the total time) and a deviation factor from the mean value is calculated.

Sharpness was chosen as a parameter because of the listeners' own response to the high frequency. The difference in sharpness is expressed in acum, and in order to calculate the value, the same algorithm is followed as for the loudness difference and the loudness growth rate. The sharpness mean value and the difference in sharpness changes over time are calculated. Then the sharpness difference distribution function is calculated and from it the $\Delta S_{5}$ value is read - the value of the sharpness difference $\Delta S_{5}$ is the level of sharpness that is "exceeded" only for $5 \%$ of the total time. Finally, the deviation factor from the mean value is calculated.

Table 3 shows these values and the final distraction level for this soundscape.

Table 3. Parameters calculation for the expressway soundscape.

\begin{tabular}{lll}
\hline Loudness difference & Mean loudness value & Deviation factor \\
\hline 3.27 & 3.94 & 0.70423 \\
Loudness growth speed difference & Mean loudness growth speed value & Deviation factor \\
1.38 & 0.4 & 3.45 \\
Sharpness difference & Mean sharpness difference value & Deviation factor \\
032 & 1.14 & 0.2807 \\
TOTAL DISTRACTION LEVEL & 0.276 & \\
\hline
\end{tabular}

\section{Conclusion}

Taking into account the game results, the questionnaire and the total distraction level, we can conclude that higher loudness is the most annoying factor. It determines general distraction and influences the distraction features of a soundscape considerably. On the other hand, loudness is not the only distraction feature and other sound characteristics must be considered. After being exposed to sudden loudness changes, the listeners adapted and the only remaining factors of distraction were the sounds perceived as different, unexpected or not corresponding to general soundscape characteristics.

In spite of adapting to a certain sound environment, providing it is not too loud, people still get distracted by unexpected sounds not "belonging" to the soundscape. A soundscape may be loud but, at the same time, not perceived as such; whereas a somewhat different sound in that soundscape, no matter how short, could be the source of distraction. This hypothesis is verified by statistical testing of the obtained results for the two groups. Using a criteria of $\alpha=0.05$, the statistical significance of the difference in average score between the two groups is affirmed and the given hypothesis is therefore proven.

The results of this testing showed that there is a distinction between annoyance and distraction, and that these two terms should be distinguished from each other in the future. Further research on this topic should focus on determining in what way particular sounds differ from their background, as well as identifying the factors that influence human perception and concentration which defines these sounds as unexpected for a given soundscape. The developed acoustic model for total distraction level (TDL) can be used in future as a quantitative measure of human distraction on any acoustic environment and can be become a helpful tool in a concept of smart cities in terms of designing a desirable and calming acoustic environments.

\section{References}

[1] JR. M. Schafer; Our Sonic Environment and the Soundscape: The Tuning of the World, Destiny Books, Vermont, (1994), p. 3-259

[2] B. Schulte-Fortkamp, A. Fiebig; Soundscape Analysis in a Residential Area: An Evaluation of Noise and People's Mind, Acta Acustica united with Acustica, Vol. 92 (2006), p. $875-880$

[3] B. Berglund and M. E. Nilsson; On a tool for measuring soundscape quality in urban residential areas, Acta Acustica united with Acustica, Vol. 92 (2006), p. 938-944

[4] E. Zwicker; Procedure for calculating loudness of temporally variable sounds, J. Acoust. Soc. Am. 62 (1977), p. 675-682

[5] E. Zwicker, H. Fastl; Psychoacoustics: Facts and models, 2nd ed. Springer-Verlag, Berlin, Heidelberg, New York (1999), p. 220

[6] U. Widmann; A psychoacoustics annoyance concept for application in sound quality, Proc. of NOISE-CON '97, (1997), p. $491-496$

[7] E. E. Ryherd, L. M. Wang; The Effects of Noise from Building Mechanical Systems with Tonal Components on Human Performance and Perception, ASHRAE Transactions, (2010), AB-10-018 (RP-1322)

[8] [L. M. Wang; Effects of Building Mechanical System Noise on Worker Performance and Perception, Proc. of NOISE-CON 2010, (2010)

[9] H. Fastl, S. Kerber, N. Guzsvány; Aspects of startling noises, Pro. of Euronoise 2009, Edinburgh (2009)

[10] J. Vos; The Loudness of Impulse and Road-Traffic Sounds in the Presence of Masking Background Noise, Acustica - Acta Acustica, Vol 84 (1998), p. 1119-1130

[11] E. Geissner, E. Parizet; Continuous Assessment of the Unpleasantness of a Sound, Acta Acustica united with Acustica, Vol. 93 (2007), p. 469-476 
[12] R. S. Schlauch; A Cognitive Influence on the Loudness of Tones That Change Continuously in Level, J. Acoust. Soc. Am., Vol. 92 (2) (1992), p. 758-765

[13] G. Canévet, B. Scharf; The Loudness of Sounds That Increase and Decrease Continuously in Level, J. Acoust. Soc. Am., Vol. 88 (5) (1990), p. 2136-2142

[14] G. Brambilla, L. Maffei; Responses to Noise in Urban Parks and in Rural Quiet Areas, Acta Acustica united with Acustica, Vol. 92 (2006), p. 881-886

[15] C. Semidor; Listening to a City With the Soundwalk Method, Acta Acustica united with Acustica, Vol. 92 (2006), p. 959-964

[16] J. Y. Jeon, P. J. Lee, J. You, J. Kang; Perceptual assessment of quality of urban soundscapes with combined noise sources and water sounds, J. Acoust. Soc. Am., Vol. 127 (2010), p. $1357-1366$

[17] P. Newbold, W. L. Carlson, B. M. Thorne; Statistics for Business and Economics, Global Edition, Eight Edition (2013)
[18] G. Milas; Istraživačke metode u psihologiji i drugim društvenim znanostima (Research Methods in Psychology and other Social Sciences), Naklada Slap, (2009), p. 91-118, $153-216$

[19] M. Mejovšek; Metode znanstvenog istraživanja u društvenim i humanističkim znanostima (Methods of Scientific Research in Social Sciences and Humanities), Naklada Slap, (2007), p. 71-158

[20] R. Larson, B. Farber; Elementary statistics: Picturing the world, Pearson Education, Inc.(2003), p. 354-482

[21] [Online]. Available:

http://www.socialresearchmethods.net/kb/stat_t.php

[22] M. Suhanek; Evaluation of soundscapes regarding sudden and unexpected sound changes, doctoral thesis, (2013) 